Pacific Journal of Mathematics

A NOTE ON MEASURES ON FOUNDATION SEMIGROUPS 


\title{
A NOTE ON MEASURES ON FOUNDATION SEMIGROUPS WITH WEAKLY COMPACT ORBITS
}

\author{
HENRY A. M. DZINOTYIWEYI AND \\ Gerard L. G. SLeiJPen
}

For an extensive class of locally compact semigroups $S$, foundation semigroups with identity element, we prove that two subalgebras of $M(S)$ [the algebra of the bounded Radon measures on $S$ ] coincide. Namely, the algebra $L(S)$, generated by the $m \in M(S)^{+}$for which the orbits on the compact subsets of $S$ are weakly compact subsets of $M(S)$, or, equivalently, for which the translations are weakly continuous, and the algebra $M_{e}(S)$, generated by the $m \in M(S)^{+}$for which the restrictions of the orbit of $m$ on $S$ to the compact subsets of $S$ are weakly compact. In case $S$ is a group, both these algebras consist of the bounded Radon measures that are absolutely continuous with respect to a Haar measure on $S$.

1. If $S$ is a locally compact group and $m \in M(S)$ then the orbits $F_{m}$ of $m$ on all compact subsets $F$ of $S\left[F_{m}:=\{m * \bar{x} \mid x \in F\} \cup\{\bar{x} * m \mid x \in F\}\right.$, where $\bar{x}$ denotes the point mass at $x]$ are weakly compact subsets of $M(S)$ if and only if the restrictions $S_{m \mid F}$ of the orbit $S_{m}$ of $m$ on $S$ to all compact subsets $F$ of $S\left[S_{m \mid F}:=\left\{\left.\mu\right|_{F} \mid \mu \in S_{m}\right\}\right]$ are weakly compact. The proof of this fact follows by observing that $F^{-1} K[:=\{x \in S \mid F x \cap K \neq \varnothing\}]$ and $K F^{-1}$ are compact as soon as both $F$ and $K$ are compact subsets of the group $S$. An arbitrary locally compact semigroup $S$ may fail to have this compactness property and may [and actually does] give rise to two different subsets of $M(S)$ : namely, to $L(S)$, the collection of all $m \in M(S)$ for which $F_{|m|}$ is weakly compact ( $F \subseteq S$, compact), and to $M_{e}(S)$, the collection of all $m \in M(S)$ for which $S_{|m| \mid F}$ is weakly compact ( $F \cong S$, compact). Elementary properties of $L(S)$ can be found in e.g., [1], [2], [6], and [7] and of $M_{e}(S)$ in [4]. Although, in some respects $M_{e}(S)$. has better properties than $L(S)$ [cf. [4], e.g., (5.2) and (5.3)] $L(S)$ is a more obvious analogue of the group algebra than $M_{e}(S): L(S)$ is a two sided $L$-ideal in $M(S)$ [cf. [1], $(3,4)$ and [2], (2.6)], while, in general, $M_{e}(S)$ is only an $L$-subalgebra of $M(S)$ [cf. (2.1) and [4], (2.6)].

It is natural to wonder about the relationship between $L(S)$ and $M_{e}(S)$. In view of the inner regularity of the measures in question, it is clear that $M_{e}(S) \subseteq L(S)$. As noted above, $M_{e}(S)$ may be strictly contained in $L(S)$, but for an important class of semigroups we can show that these collections coincide. We shall prove the following theorem. 
THEOREM 1.1. Let $S$ be a locally compact [topological] semigroup with identity element 1 such that for all compact neighborhoods $U$ of 1 we have that

(i ) $x \in \operatorname{int}\left(U^{-1}(U x) \cap(x U) U^{-1}\right)(x \in S)$ and

(ii) $1 \in \operatorname{int}\left(U^{-1} v \cap u U^{-1}\right)$ for some $u, v \in U$. Then $L(S)=M_{e}(S)$.

The semigroup $S$, in the theorem, is a so-called stip [cf. [7], (2.1)]. The class of stips is extensive, contains the foundation semigroups $S$ [i.e., $\cup\{\operatorname{supp}(m) \mid m \in L(S)\}$ is dense in $S$ ] with identity element [cf. [7], (2.2)] and, furthermore, contains many semigroups $S$ that contain compact subsets $F$ and $K$ of $S$ such that $F^{-1} K$ is not compact [cf. [6], App. B].

The notations and conventions that are not explained in the text or the introduction are the ones of [7].

2. The failure of $M_{e}(S)$; an example. Before we proceed to prove the theorem, we give an example of a foundation semigroup $S$ for which $M_{e}(S)$ is not an ideal in $M(S)$, and, consequently, $M_{e}(S) \varsubsetneqq L(S)$. This example solves the question (2.7) in [4].

EXAMPLE 2.1. Let $T_{1}$ be the subset $[0,1]_{d} \times\{0\} \cup[0,1] \times\{1\} \cup\{\theta\}$ of $\boldsymbol{R}^{2}$, where $\theta$ is the point $(1,2)$ in $\boldsymbol{R}^{2} . \quad[0,1]_{d} \times\{0\}$ is endowed with the discrete topology and $[0,1] \times\{1\} \cup\{\theta\}$ with the restriction topology of the usual topology on $\boldsymbol{R}^{2}$. Then $T_{1}$ is a locally compact semigroup under the multiplication defined as follows:

$$
\left.\begin{array}{l}
\left(t, t^{\prime}\right) \theta:=\theta\left(t, t^{\prime}\right):=\theta \theta:=\theta \\
\left(t, t^{\prime}\right)\left(s, s^{\prime}\right):=\left(t, s^{\prime}\right) \text { if } t^{\prime}=0 \\
\left(t, t^{\prime}\right)\left(s, s^{\prime}\right):=\theta \text { if } t^{\prime} \neq 0 .
\end{array}\right\} \text { for all }\left(t, t^{\prime}\right),\left(s, s^{\prime}\right) \in T_{1} \backslash\{\theta\}
$$

Now, let $a$ be an element isolated from $T_{1}$. Put

$$
T:=T_{1} \cup\{a\} \text {. }
$$

And let the multiplication on $T_{1}$ be extended to $T$ by:

$$
\left.\begin{array}{l}
a a:=a, a \theta:=\theta a:=\theta \\
a\left(t, t^{\prime}\right):=\left(0, t^{\prime}\right) \\
\left(t, t^{\prime}\right) a:=(t, 0) \text { if } t^{\prime}=0 \\
\left(t, t^{\prime}\right) a:=\theta \text { if } t^{\prime} \neq 0 .
\end{array}\right\} \text { for all }\left(t, t^{\prime}\right) \in T \backslash\{\theta\}
$$

Then one can easily check that $T$ is a locally compact semigroup [only the associativity needs some real verification], and that $T$ is even a foundation semigroup. 
Furthermore, one has that

$$
\begin{aligned}
& \{\bar{a} * \bar{x} \mid x \in T\}=\{\bar{a}, \bar{\theta}, \overline{(0,1)}, \overline{(0,0)}\} \\
& \{\bar{x} * \bar{a} \mid x \in T\}=\{\bar{a}, \bar{\theta}\} \cup\{\overline{(t, 0)} \mid t \in[0,1]\} .
\end{aligned}
$$

Apparently, $\bar{a} \in M_{e}(T)$. However, $\bar{a} * \overline{(0,1)}=\overline{(0,1)}$ and $\overline{(0,1)} \notin M_{e}(T)$; because $\{\overline{(t, 0)} * \overline{(0,1)} \mid t \in \overline{[0,1]}\}=\{\overline{(t, 1)} \mid t \in[0,1]\}$. This shows that $M_{e}(T)$ is not a right ideal in $M(T)$.

Let $b$ be an element, isolated from $T \times\{1,2\}$. Put $S:=T \times\{1,2\} \cup(b)$ and let the multiplication on $S$ be defined by

$$
\left.\begin{array}{rl}
s b:=b s: & =b \text { for all } s \in S \\
(x, 1)(y, 2): & =(y, 2)(x, 1):=b \\
(x, 1)(y, 1): & =(x y, 1),(x, 2)(y, 2):=(y x, 2)
\end{array}\right\} \text { for all } x, y \in T .
$$

Then $S$ is a foundation semigroup and $M_{e}(S)$ is neither a right nor a left ideal in $M(S)$.

3. Preliminaries to the proof of the theorem. Before we proceed to the proof of theorem we prove a lemma that can be viewed as a tool to reduce the "underlying space" to a space satisfying the second axiom of countability [cf. (3.4)]. For the convenience of the reader we first summarize some properties concerning the structure of a stip $S$ and its "semigroup algebra" $L(S)$ [cf. (3.1)-(3.3)].

Proposition 3.1 [cf. [7], (2.4), (2.7), and (2.11).] Let $S$ be a stip. Then $S$ has a smallest dense ideal $\dot{S}$ [i.e., $S S \cup \dot{S} S \subseteq \dot{S}$, clo $(\dot{S})=S$ and $\dot{S} \subseteq J$ for all $J \subseteq S$ with $\bar{J}=S$ and $J S \subseteq J$ or $S J \subseteq J]$.

For each pair of open subset $W$ and $U$ of $S$ and each $x \in \dot{S}$ the sets $W^{-1}(U x),(x U) W^{-1},\left(W \cap S^{-1} x\right.$ and $x\left(W \cap S^{-1}\right.$ are open. For each neighborhood $U$ of 1 there exists a neighborhood $V$ of 1 such that

$$
\widetilde{U}:=\{(x, y) \in S \times S \mid U x \cap U y \neq \varnothing\} \supseteqq \widetilde{V} \circ \widetilde{V}
$$

[where $A \circ B:=\{(x, z) \in S \times S \mid(x, y) \in A, \quad(y, z) \in B$ for some $y \in S\}$, $(A, B \subseteq S \times S)]$.

Proposition 3.2 [cf. [7]. (3.6) and (3.7)].

Let $S$ be a stip. An idempotent $e$ in $S$ [i.e., ee $=e$ ] is said to be $\delta$-isolatd if there exists a $G_{\delta}$-set $G$ in $S$ for which $(G \cap e S e) \backslash\{e\}$ contains no idempotents.

Then for each countable subset $A$ of $\dot{S}$ and each $\mu \in L(S)$ with compact support there exists a $\delta$-isolated idempotent $e$ such that 


$$
\operatorname{supp}(\mu) \cup A \subseteq e S e
$$

Proposition 3.3 [cf. [1], (3.4), [6], (2.6) and [7], (3.13)].

(1) For each $\mu \in M(S)$, the maps $x m \bar{x} * \mu(x \in S)$ and $x m \mu * \bar{x}$ $(x \in S)$ from $S$ into $M(S)$ are weakly* continuous. Let $S$ be a stip.

(2) Then $L(S)$ is an L-ideal in $M(S)$.

(3) For $\mu \in M(S)$, the following properties are equivalent:

(i) $\mu \in L(S)$;

(ii) the map $x m \bar{x} * \mu$ from $S$ into $M(S)$ is norm continuous;

(iii) the map $x m \mu * \bar{x}$ from $S$ into $M(S)$ is norm continuous.

(4) If, in addition, $S$ is a foundation semigroup then, for all $\mu \in L(S)$ and $\varepsilon>0$, there exists a $\nu \in L(S)$ such that $\|\nu\|=\nu(S)=1$ and

$$
\|\nu * \mu-\mu\|<\varepsilon .
$$

Now we can prove the lemma.

LEMMA 3.4. Let $S$ be a stip with a $\delta$-isolated identity element 1 , let $\left(V_{n}\right)_{n \in N}$ be a sequence of neighborhoods of 1 and let $M$ be a compact subset of $S$.

Then there exists a decreasing sequence $\left(U_{n}\right)_{n \in N}$ of open relativelycompact neighborhoods of 1 and a semi-metric $\rho$ on $S$ such that

(1) $\quad U_{n+1}^{-1}\left(U_{n+1} x\right) \subseteq B_{\rho}(x, 1 / n) \subseteq U_{n}^{-1}\left(U_{n} x\right) \quad(n \in N, x \in S)$, uhere $B_{\rho}(x, \varepsilon):=\{y \in S \mid \rho(x, y)<\varepsilon\} \quad(x \in S, \varepsilon>0)$;

(2) $G:=B_{\rho}(1,0)$ is a compact group with identity element 1 contained in $V_{n}$ for all $n \in N$.

Let $\pi$ be the normalized Haar measure on $G$. Then

(3) for each $\sigma$-compact subset $A$ of $S, P:=\bigcap_{m=1}^{\infty} U_{m} A$ is a Borel set and $|\pi * \mu|(\bar{P} \backslash P)=0$ for all $\mu \in L(S)$.

Finally, there exists a countable subset $T$ of $S$ such that

(4) for all $x \in M$ and all neighborhood $W$ of 1 we have that $G W x \cap T \neq \varnothing$.

Proof. Since 1 is $\delta$-isolated there exists a sequence $\left(U_{n}^{\prime}\right)_{n \in N}$ of neighborhoods of 1 such that $\bigcap_{n=1}^{\infty} U_{n}^{\prime} \backslash\{1\}$ contains no idempotents. Now, by induction and using (3.1), one can construct a decreasing sequence $\left(U_{n}\right)_{n \in N}$ of open relatively-compact neighborhoods of 1 and a sequence $\left(r_{n}\right)_{n \in N}$ of elements of $\dot{S}$ such that for all $n \in N$ we have $r_{n} \in U_{n}$

(i ) $\quad \bar{U}_{n+1} \subseteq U_{n} \cap U_{n}^{\prime} \cap V_{n} \cap U_{n}^{-1} r_{n}$

(ii) $U_{n+1}^{2} \subseteq U_{n}$

(iii) $\widetilde{U}_{n+1} \circ \widetilde{U}_{n+1} \circ \widetilde{U}_{n+1} \subseteq \widetilde{U}_{n}$.

From (iii) and the result on p. 184-186 in [5] [see also [7], (2.13)] 
it follows that there exists a semi-metric $\rho$ with property (1). By using (i) and (ii), one can easily conclude that (2) holds.

To prove (3), let $\left(F_{n}\right)_{n \in \mathbf{N}}$ be a sequence of compact subsets of $S$. Put $A:=\bigcup_{n=1}^{\infty} F_{n}$ and $P:=\bigcap_{m=1}^{\infty} U_{m} A$. Then by (i) we have

$$
P=\bigcap_{m=1}^{\infty} U_{m} A \subseteq \bigcap_{m=1}^{\infty} \bar{U}_{m} A \subseteq \bigcap_{m=2}^{\infty} U_{m-1} A=P .
$$

Since $\bar{U}_{m} A=\bigcup_{n=1}^{\infty} \bar{U}_{m} F_{n}$ is $\sigma$-compact $(m \in N)$, this proves that $P$ is a Borel set. Furthermore, by (1), (i) and (ii), we have that

$$
\begin{aligned}
& \bar{P} \subseteq B_{\rho}(P, 1 /(n+4)) \subseteq U_{n+3}^{-1}\left(U_{n+3} P\right) \\
& \cong r_{n+2}^{-1}\left(U_{n+1}\left(U_{n+1} A\right)\right) \cong r_{n+2}^{-1}\left(U_{n} A\right) \leqq r_{n+2}^{-1}\left(\bar{U}_{n} A\right) \quad(n \in N) .
\end{aligned}
$$

Now let $\varepsilon>0$ and $\mu \in L(S)$. Then, by (3.3.3), we have that

$$
V:=\{x \in S|||\bar{x} * \pi *| \mu|-\pi *| \mu|| \mid<\varepsilon\}
$$

is an open neighborhood of $G$. Since $G$ is a compact group, (2) implies that there exists a $k \in N$ such that

$$
U_{k} \subseteq V \text {. }
$$

We may assume that

$$
|\pi *| \mu|(P)-\pi *| \mu\left|\left(\bar{U}_{k} A\right)\right|<\varepsilon .
$$

Obviously, $r_{k+2} \in U_{k} \subseteq V$, which leads to

$$
\begin{aligned}
& \pi *|\mu|\left(\bar{U}_{k} A\right)-\varepsilon \leqq \pi *|\mu|(P) \leqq \pi *|\mu|(\bar{P}) \\
& \bar{r}_{k+2} * \pi *|\mu|\left(\bar{U}_{k} A\right) \leqq \pi *|\mu|\left(\bar{U}_{k} A\right)+\varepsilon .
\end{aligned}
$$

This shows that $|\pi * \mu|(\bar{P} \backslash P) \leqq \pi *|\mu|(\bar{P} \backslash P) \leqq 2 \varepsilon$, and completes the proof of (3).

To prove $(4)$, define the equivalence relation $\approx$ on $S$ by

$$
x \approx y \quad \text { if and only if } \rho(x, y)=0 \quad(x, y \in S) .
$$

Put $\tilde{\widetilde{x}}:=\{y \in S \mid \rho(x, y)=0\}, \quad \widetilde{B}:=\{\widetilde{\tilde{b}} \mid b \in B\} \quad(x \in S, B \leqq S)$ and let $\Sigma$ be the quotient space $S / \approx$. Then $\Sigma$ is a metric space and $\widetilde{\widetilde{M}}$ is a compact subset of $\Sigma$. Therefore we can find a countable subset $A$ of $S$ such that

$$
\operatorname{clo}(\tilde{\widetilde{A}})=\tilde{\widetilde{M}} \text { in } \Sigma \text {. }
$$

Define $T:=\left\{r_{n} a \mid n \in N, a \in A\right\}$. We shall prove that this set $T$ has property (4). Obviously $T$ is countable. Let $W$ be an open neighborhood of 1 and let $x \in M$. Since $G$ is a compact group, $G W$ is an 
open neighborhood of $G$ and, by (2), there exists a $k \in N$ such that $U_{k} x \subseteq G W x$. Furthermore we have that

$$
\begin{gathered}
B_{\rho}(\widetilde{\widetilde{x}}, 1 /(k+2))=B_{\rho}(x, 1 /(k+2)) \\
\leqq U_{k+2}^{-1}\left(U_{k+2} x\right) \cong r_{k+2}^{-1}\left(U_{k} x\right) .
\end{gathered}
$$

Since $\widetilde{\widetilde{A}}$ is dense in $\widetilde{\widetilde{M}}$ it follows that $B_{\rho}(\widetilde{\widetilde{x}}, 1 /(k+2)) \cap \widetilde{\widetilde{A}} \neq \varnothing$ and therefore $G W x \cap T \neq \varnothing$.

4. The proof of theorem. Initially, we shall prove the theorem for a foundation semigroup $S$ with identity element. Then we shall indicate how to prove the theorem for stips.

(A) Let $S$ be a foundation semigroup with identity element 1 and let $m \in L(S)$.

To prove that $m \in M_{e}(S)$, we may assume without loss of generality that $M:=\operatorname{supp}(m)$ is compact, $m \geqq 0$ and $\|m\|=1$. Take a compact subset $K$ of $S$. In view of (3.3.1), it is sufficient to show that $\left\{\left.(m * \bar{x})\right|_{K} \mid x \in S\right\}$ is a relatively weakly compact subset of $M(S)$. A combination of the theorem of Eberlein and Smulian [cf. e.g., [3], V. 6.1], (3.3.1) and the denseness of $S$ in $S$ tells us now that $m \in M_{e}(S)$ as soon as

$$
\left\{\begin{array}{l}
\left\{\left.\left(m * \bar{x}_{n}\right)\right|_{K} \mid n \in N\right\} \text { has a weak limit point in } M(S) \\
\text { for all sequences }\left(x_{n}\right)_{n \in N} \text { in } \dot{S} .
\end{array}\right.
$$

Let $\left(x_{n}\right)_{n \in N}$ be a sequence in $\dot{S}$. By (3.2), there exists a $\delta$-isolated idempotent $e$ such that $M \cup\left\{x_{n} \mid n \in N\right\} \subseteq e S e$. Therefore, again without loss of generality, we may assume that 1 is $\delta$-isolated.

For each $n \in N$ let $V_{n}:=\{x \in S \mid\|\bar{x} * m-m\|<1 / n\}$. Then $\left(V_{n}\right)_{n \in,}$ is a sequence of neighborhoods of 1 [cf. (3.3.3)] and we can apply our lemma. In the sequel, we shall use the same notations as in the lemma and its proof.

Since $G \subseteq V_{n}(n \in N)$ it follows that

$$
\bar{g} * m=m(g \in G),
$$

and hence that

$$
\pi * m=m \text {. }
$$

Let $\left\langle t_{1}, t_{2}, t_{3}, \cdots\right\rangle$ be an enumeration of $T$.

Now, by induction, one can construct sequences

$x_{1}^{0}, x_{2}^{0}, x_{3}^{0}, x_{4}^{0}, \cdots$

$x_{1}^{1}, x_{2}^{1}, x_{3}^{1}, x_{4}^{1}, \cdots$

$x_{1}^{2}, x_{2}^{2}, x_{3}^{2}, x_{4}^{2}, \cdots$

$$
\text { ....... }
$$


with the following properties

(a) $x_{m}^{0}=x_{m}(m \in N)$,

(b) $\left(x_{m}^{n+1}\right)_{m \in N}$ is a subsequence of $\left(x_{m}^{n}\right)_{m \in \mathbf{N}}(n \in N)$,

(c) $\left(t_{m} x_{m}^{n}\right)_{m \in N}$ is a $\rho$-Cauchy sequence or

$x_{m}^{n}=x_{m}^{n-1}(m \in N)$ and $\left(t_{n} x_{m}^{n}\right)_{m \in \mathbf{N}}$ does not contain any $\rho$-Cauchy sequence $(n \in N)$.

Put $y_{m}:=x_{m}^{m}(m \in N), T_{1}:=\left\{t \in T \mid\left(t y_{m}\right)_{m \in N}\right.$ is a $\rho$-Cauchy sequence $\}$, $P:=\bigcap_{m=1}^{\infty} U_{m} T_{1}, m_{1}:=\left.m\right|_{P}$ and $m_{2}:=m-m_{1}$.

We shall prove that

( $\alpha)\left(\pi * m_{1} * \bar{y}_{n}\right)_{n \in N}$ is a norm-Cauchy sequence in $M(S)$, and

( $\beta) \quad\left(\left.\left(\pi * m_{2} * \bar{y}_{n}\right)\right|_{K}\right)_{n \in N}$ converges in norm to 0 ;

then obviously $\left(\left.\left(\pi * m * \bar{y}_{n}\right)\right|_{K}\right)_{n \in N}$ is a Cauchy sequence and consequently $\left\{\left.\left(\pi * m * \bar{x}_{n}\right)\right|_{K} \mid n \in N\right\}$ has a weak limit point. Therefore, in view of (2), this proves (1) and completes the proof of the theorem in this case.

( $\alpha$ ) Let $\varepsilon>0$. By (3.3.4), there exists a $\nu \in L(S)^{+}$such that

$$
\left\|\nu * \pi * m_{1}-\pi * m_{1}\right\|<\varepsilon / 4 \text {. }
$$

Since $\{x \in S \mid\|\nu * \pi * \bar{x}-\nu * \pi\|<\varepsilon / 4\}$ is a neighborhood of $G$ [cf. (3.3)], there exists a $k \in N$ such that

$$
\bar{U}_{k} \subseteq\{x \in S \mid\|\nu * \pi * \bar{x}-\nu * \pi\|<\varepsilon / 4\}
$$

and

$$
\left\|m_{1}-\left.m\right|_{\overparen{i j}_{k} T_{1}}\right\|<\varepsilon / 8 \text {. }
$$

Furthermore, there exists a finite subset $E$ of $T_{1}$ such that

$$
\left\|\left.m\right|_{\bar{U}_{k} T_{1}}-\left.m\right|_{\bar{U}_{k} E}\right\|<\varepsilon / 8 .
$$

Since $\left(t y_{n}\right)_{n \in N}$ is a $\rho$-Cauchy sequence for all $t \in E$, we can find an $l \in N$ such that

$$
U_{k} t y_{n} \cap U_{k} t y_{m} \neq \varnothing \quad(n, m \geqq l, t \in E) \quad[\text { cf. (3.4.1)] . }
$$

Now, by (4), we have that

$$
\left\|\nu * \pi *(\overline{x t}) * \bar{y}_{n}-\nu * \pi *(\overline{x t}) * \bar{y}_{m}\right\|<\varepsilon \quad\left(n, E>l, x \in \bar{U}_{k}, t \in E\right) .
$$

Therefore,

$$
\left\|\left.\nu * \pi * m\right|_{\bar{\iota}_{k}} U * \bar{y}_{n}-\left.\nu * \pi * E\right|_{\bar{v}_{k} E} * \bar{y}_{m}\right\|<\varepsilon \quad(n, m \geqq l) .
$$

Now, an application of (3), (5), and (6) leads to

$$
\left\|\pi * m_{1} * \bar{y}_{n}-\pi * m_{1} * \bar{y}_{m}\right\|<3 \varepsilon / 2 \quad(n, m \geqq l) .
$$

This shows that 
$\left(\pi * m_{1} * \bar{y}_{n}\right)_{n \in N}$ is a Cauchy sequence in $M(S)$.

( $\beta$ ) For each $n \in N$, let $P_{n}:=\left\{x \in S \mid x y_{m} \notin G K(m \in N, m \geqq n)\right\}$. We shall show that

$$
M \backslash \bar{P} \subseteq \bigcup_{n=1}^{\infty} P_{n} .
$$

For this purpose, suppose these exists an $x \in M \backslash \bar{P}$ such that

$$
x \notin P_{n} \text { for all } n \in N \text {. }
$$

Then there is an open neighborhood $W$ of $x$ such that $W \cap P=\varnothing$. Since $W x^{-1}$ is a neighborhood of $1,(3.4 .4)$ tells us that $G\left(W x^{-1}\right) x \cap$ $T \neq \varnothing$. Therefore, there are $g \in G, v \in W x^{-1}$ and $t \in T$ such that $g^{-1} t=v x \in W$. Hence $g^{-1} t \notin P$ or $t \notin g P=g\left(\bigcap_{m=1}^{\infty} U_{m} T_{1}\right)=\bigcap_{m=1}^{\infty} g U_{m} T_{1} \subseteq$ $\bigcap_{m=2}^{\infty} U_{m-1} T_{1}=P$. In particular, we have that $t \notin T_{1}$. Since $x \notin P_{n}$ $(n \in N)$, one can find a subsequence $\left(z_{n}\right)_{n \in N}$ of $\left(y_{n}\right)_{n \in N}$ such that $x z_{n} \in G K(n \in N)$. This implies that $t z_{n}=g v x z_{n} \in g v G K(n \in N)$, or in other words, $\left(t z_{n}\right) \approx \in(g v G K) \approx(n \in N)$. However, this violates the fact that $t \notin T_{1}$; because $(g v G K) \approx$ is a compact subset of the metric space $\Sigma$. Apparently, (7) holds.

Now let $\varepsilon>0$. Recall that $m(\bar{P})=m(P)$ [cf. (3.4.3)]. Hence, by (7), we can find a $k \in N$ such that

$$
\left\|m_{2}-\left.m_{2}\right|_{P_{k}}\right\|<\varepsilon \text {. }
$$

Since $g x y_{n} \notin K$ for all $g \in G, n \geqq k, x \in P_{k}$, we also have that

$$
\left.\pi * m_{2}\right|_{P_{k}} * \bar{y}_{n}(K)=0 \text { for all } n \geqq k \text {. }
$$

A combination of this property with the previous one leads to

$$
\begin{aligned}
& \left\|\left.\left(\pi * m_{2} * \bar{y}_{n}\right)\right|_{K}\right\| \\
& \quad \leqq\left\|\left.\left(\pi * m_{2} * \bar{y}_{n}\right)\right|_{K}-\left.\left(\left.\pi * m_{2}\right|_{P_{k}} * \bar{y}_{n}\right)\right|_{K}\right\|+\left(\left.\pi * m_{2}\right|_{P_{k}} * \bar{y}_{n}\right)(K) \leqq \varepsilon \quad(n \geqq k) .
\end{aligned}
$$

This completes the proof of $(\beta)$ and hence the proof of the theorem for foundation stips.

(B) Finally, let $S$ be a stip.

Note that, to prove the theorem in this case, it is sufficient to adjust the above proof of $(\alpha)$. One can do this by using techniques as developed in [7], (3.4)-(3.8). Since the difficulties, by using these methods, are even more technical than the ones in the already presented proofs, we shall restrict ourselves to an indication of this proof.

For each $x \in S$, let $\cdot{ }_{x}$ be the map from $S x \times x S$ into $S$, defined by

$$
p x \cdot{ }_{x} x q:=\cdot_{x}(p x, x q):=p x q \quad(p, q \in S) .
$$


These maps $\cdot_{x}(x \in S)$ induce "convolution products" $*_{x}$ between measures $\nu \in L(S)$ with $\nu \in M(S x)$ [i.e., $|v|(S \backslash A)=0$ for a certain $\sigma$ compact subset $A$ of $S x]$ and $\mu \in L(S)$ with $\mu \in M(x S)$ by

$$
\nu *_{x} \mu(f):=\iint f\left(y \cdot{ }_{x} z\right) d \nu(y) d \mu(z) \quad\left(f \in C_{\infty}(S)\right) ;
$$

note that the fact that $S$ is a stip implies that $|\nu|(S \backslash A x)=$ $|\mu|(S \mid x A)=0$ for a certain $\sigma$-compact subset $A \subseteq S$ [see also [7], (3.4)]. As in (3.3.2) one has for the above $\nu$ and $\mu$ that $\nu *_{x} \mu \in L(S)$ and also that $\nu *_{x}(\mu * \rho)=\left(\nu *_{x} \mu\right) * \rho(\rho \in M(S))$. Furthermore, one has that for each $x \in d(S):=\operatorname{clo} \cup\{\operatorname{supp}(\mu) \mid \mu \in L(S)\}, \varepsilon>0$ and $\mu \in L(S)$ there exists a $\nu \in L(S)^{+}$with $\|\nu\|=\nu(S x)=1$ such that

$$
\left\|\left.\mu\right|_{x S}-\left.\nu *_{x} \mu\right|_{x S}\right\|=\left.|| \bar{x} *_{x} \mu\right|_{x S}-\left.\nu *_{x} \mu\right|_{x S} \|<\varepsilon .
$$

Using these results and an adjusted group $G$, one obtain, by changing the proof of $(\alpha)$ in the obvious ways, that

$$
\left(\left.\left(\pi * m_{1}\right)\right|_{x S} * \bar{y}_{n}\right)_{n \in N} \text { is a Cauchy sequence in } M(S) \quad(x \in d(S)) .
$$

As is proved implicitly in $\S 3$ of [7], there exists a sequence $\left(x_{n}\right)_{n \in N}$ in $d(S)$ such that

$$
\left.\pi * m_{1} \ll \sum_{n=1}^{\infty} 2^{-n}\left(\pi * m_{1}\right)\right|_{x_{n} S} .
$$

Using (3.5) of [6], this leads to the relatively weak compactness of $\left\{\pi * m_{1} * \bar{y}_{n} \mid n \in N\right\}$.

\section{REFERENCES}

1. Anne C. Baker and J. W. Baker, Algebras of measures on a locally compact semigroup, J. London Math. Soc., 2 (1970), 651-659.

2. - Algebras of measures on a locally compact semigroup III, J. London Math. Soc., 4 (1972), 685-695.

3. N. Dunford and J. Schwartz, Linear Operators, Part I: General Theory, Interscience, New York, 1966.

4. Henry A. M. Dzinotyiweyi, On the analogue of the group algebra for locally compact semigroups, J. London Math. Soc., 17 (1978), 489-506.

5. J. L. Kelley, General Topology, Van Nostrand, New York, 1955.

6. Gerard L. G. Sleijpen, Convolution measure algebras on semigroups, Thesis, Catholic University, Toernooiveld, Nijmegen, 1976.

7. - Locally compact semigroups and continuous translation of measures, Proc. London Math. Soc., 37 (1978), 75-97.

Received February 15, 1978. The work of the second author was partially supported by the Netherlands Organization of Pure Research, Z.W.O.

UNIVERSITY OF OREGON

EUGENE, OR 97405

AND

UNIVERSITY OF ABERDEEN

ABERDEEN AB9 2TY, U.K. 



\section{PACIFIC JOURNAL OF MATHEMATICS}

EDITORS

DONALD BABBITT (Managing Editor)

University of California

Los Angeles, California 90024

HUGo RossI

University of Utah

Salt Lake City, UT 84112

C. C. MOORE

J. DUGUNDJI

Department of Mathematics

University of Southern California

Los Angeles, California 90007

R. FinN AND J. MiLgraM

Stanford University

Stanford, California 94305

University of California

Berkeley, CA 94720

\section{ASSOCIATE EDITORS}

E. F. BrCKENBACH

B. H. NeUmanN

F. WOLF

K. YoshidA

\section{SUPPORTING INSTITUTIONS}

UNIVERSITY OF BRITISH COLUMBIA

UNIVERSITY OF SOUTHERN CALIFORNIA

CALIFORNIA INSTITUTE OF TECHNOLOGY

STANFORD UNIVERSITY

UNIVERSITY OF CALIFORNIA

UNIVERSITY OF HAWAII

MONTANA STATE UNIVERSITY

UNIVERSITY OF TOKYO

UNIVERSITY OF NEVADA, RENO

UNIVERSITY OF UTAH

NEW MEXICO STATE UNIVERSITY

WASHINGTON STATE UNIVERSITY

OREGON STATE UNIVERSITY

UNIVERSITY OF WASHINGTON

UNIVERSITY OF OREGON 


\section{Pacific Journal of Mathematics}

\section{Vol. 81, No. $1 \quad$ November, 1979}

Thomas E. Armstrong, Simplicial subdivision of infinite-dimensional compact cubes ..................................... 1

Herbert Stanley Bear, Jr., Approximate identities and pointwise convergence ................................

Richard David Bourgin, Partial orderings for integral representations on convex sets with the Radon-Nikodým property..................

Alan Day, Herbert S. Gaskill and Werner Poguntke, Distributive lattices

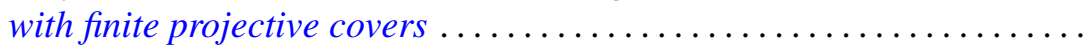

Heneri Amos Murima Dzinotyiweyi and Gerard L. G. Sleijpen, A note on measures on foundation semigroups with weakly compact orbits ......

Ronald James Evans, Resolution of sign ambiguities in Jacobi and Jacobsthal sums ...................................

John Albert Fridy, Tauberian theorems via block dominated matrices ......

Matthew Gould and Helen H. James, Automorphism groups retracting onto

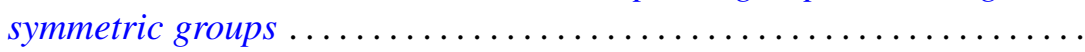

Kurt Kreith, Nonlinear differential equations with monotone solutions . . . . 101

Brian William McEnnis, Shifts on indefinite inner product spaces........ 113

Joseph B. Miles, On entire functions of infinite order with radially

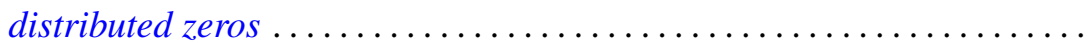

Janet E. Mills, The idempotents of a class of 0-simple inverse semigroups ...............................

Edward Jean Moulis, Jr., Generalizations of the Robertson functions ...

Richard A. Moynihan and Berthold Schweizer, Betweenness relations in probabilistic metric spaces.......................

Stanley Ocken, Perturbing embeddings in codimension two ....

Masilamani Sambandham, On the average number of real zeros of a class of random algebraic curves.

Jerry Searcy and B. Andreas Troesch, A cyclic inequality and a related eigenvalue problem.

Roger R. Smith and Joseph Dinneen Ward, $M$-ideals in $B\left(l_{p}\right)$...

Michel Talagrand, Deux généralisations d'un théorème de I. Namioka ..

Jürgen Voigt, $O n Y$-closed subspaces of $X$, for Banach spaces $X \subset Y$;

existence of alternating elements in subspaces of $C(J)$

Sidney Martin Webster, On mapping an $n$-ball into an $(n+1)$-ball in complex spaces

David J. Winter, Triangulable subalgebras of Lie p-algebras ... 